\title{
Capacity and Delay of Hybrid Wireless Broadband Access Networks
}

\author{
Pan Li, Chi Zhang, Student Member, IEEE, and Yuguang Fang, Fellow, IEEE
}

\begin{abstract}
An optical network is too costly to act as a broadband access network. On the other hand, a pure wireless ad hoc network with $n$ nodes and total bandwidth of $W$ bits per second cannot provide satisfactory broadband services since the pernode throughput diminishes as the number of users goes large. In this paper, we propose a hybrid wireless network, which is an integrated wireless and optical network, as the broadband access network. Specifically, we assume a hybrid wireless network consisting of $n$ randomly distributed normal nodes, and $m$ regularly placed base stations connected via an optical network. A source node transmits to its destination only with the help of normal nodes, i.e., in the ad hoc mode, if the destination can be reached within $L(L \geq 1)$ hops from the source. Otherwise, the transmission will be carried out in the infrastructure mode, i.e., with the help of base stations. Two transmission modes share the same bandwidth of $W$ bits/sec. We first study the throughput capacity of such a hybrid wireless network, and observe that the throughput capacity greatly depends on the maximum hop count $L$ and the number of base stations $m$. We show that the throughput capacity of a hybrid wireless network can scale linearly with $n$ only if $m=\Omega(n)$, and when we assign all the bandwidth to the infrastructure mode traffics. We then investigate the delay in hybrid wireless networks. We find that the average packet delay can be maintained as low as $\Theta(1)$ even when the per-node throughput capacity is $\Theta(W)$.
\end{abstract}

Index Terms-Hybrid wireless networks; capacity; delay; broadband access networks.

\section{INTRODUCTION}

$\mathbf{T}$ HE INTERNET'S phenomenal growth has triggered great increase on demands for broadband services. Thus, how to design a broadband access network to provide broadband services is essential to the further success of the Internet. Optical networks can provide high bandwidth and low network delay [3] [25] [26]. However, they are too costly to act as broadband access networks. Since wireless networks can be deployed easily and quickly with low cost, we then turn to them for help.

In their seminal paper [12], Gupta and Kumar show that the per-node throughput capacity in random wireless ad hoc networks is $\Theta\left(\frac{W}{\sqrt{n \log n}}\right)^{1}$ bits/sec, which means random ad hoc networks cannot scale. Later on, Buragohain et al. [4] study

Manuscript received 15 January 2008; revised 4 July 2008. This work was partially supported by the National Science Foundation under grants CNS0721744 and DBI-0529012. The work of Fang was also partially supported by the 111 Project under B08038 with Xidian University, China.

P. Li, C. Zhang and Y. Fang is with Department of Electrical and Computer Engineering, University of Florida, Gainesville, FL 32611 (e-mail: \{lipanleo@,zhangchi@,fang@ece.\}ufl.edu).

Y. Fang is also a Changjiang Scholar Chair Professor with National Key Laboratory of Integrated Services Networks, Xidian University, Xi'an, China.

Digital Object Identifier 10.1109/JSAC.2009.090203.

${ }^{1}$ In this paper, we use the Knuth's notations [13]: $f(n)=O(g(n))$ means $f(n)$ is asymptotically upper bounded by $g(n) ; f(n)=\Omega(g(n))$ means $f(n)$ is asymptotically lower bounded by $g(n) ; f(n)=\Theta(g(n))$ means $f(n)$ is asymptotically tight bounded by $g(n) ; f(n)=o(g(n))$ means $f(n)$ is asymptotically negligible with respect to $g(n) ; f(n)=\omega(g(n))$ means $f(n)$ is asymptotically dominant with respect to $g(n)$. the throughput capacity in grid networks where there are $n$ nodes and the average source-destination distance is $d$. They show that the $\Omega(n / d)$ throughput can be achieved. Thus, grid networks cannot scale either since $d=\omega(1)$ for most of the cases.

The work in [12] deals with dense networks, i.e., the area is fixed and the node density increases linearly as the number of nodes, and the authors assume the whole network is connected. Dousse et al. [6] study the throughput capacity in extended networks where the density of nodes is fixed and the area increases linearly with the number of nodes $n$. They show that by allowing an arbitrary small fraction of the nodes to be disconnected in 2-dimensional extended networks, a nonvanishing rate can be achieved for each node. Ozgur et al. [22] also investigate the throughput capacity of a connected ad hoc network. Their results show that by intelligent node cooperation and distributed MIMO communication, the dense networks can scale linearly with the number of nodes $n$, and the extended networks scale as $n^{2-\alpha / 2}$ for $2 \leq \alpha<3$ and $\sqrt{n}$ for $\alpha \geq 3$, where $\alpha$ is the path loss exponent in power propagation model. Moreover, Duarte-Melo et al. [7] study the case of semi-extended networks, where both node density and the network area increase as the number of nodes $n$ increases. Specifically, they assume the network area is a disk of radius $n^{\gamma}, 0<\gamma<\frac{1}{2}$. With a $\frac{1}{(1+d)^{\alpha}}$ propagation model, they show that the per-node throughput capacity is $\Omega\left(\frac{1}{n^{1-\gamma}}\right)$, i.e., semiextended networks cannot scale.

Since we prefer the network to be connected, and the nodes in the network to be loosely coupled as well, the results for pure ad hoc networks are pessimistic, i.e., they cannot scale as the number of nodes. This means pure wireless ad hoc networks cannot provide satisfactory broadband service when the number of network users goes large. In this study, we propose to use hybrid wireless networks as the broadband access networks, which are also called multihop cellular networks [18]. Hybrid wireless networks can be one-dimensional, two-dimensional with strip area [17], or two-dimensional with square area [16]. Traffic pattern in the network can be asymmetric [27] or symmetric [14] [23] [28]. In this study, we only focus on two-dimensional square hybrid wireless networks with symmetric traffics.

We first investigate the throughput capacity of hybrid wireless networks. Kozat and Tassiulas [14] study the throughput capacity of hybrid wireless networks where both ad hoc nodes and access points are randomly distributed. They show that the per-node throughput capacity can be $\Theta(W / \log n)$ bits per second if the number of access points scales linearly with the number of nodes, which means the network cannot scale. Similar results are also reported in [1]. Zemlianov and Veciana [28] investigate the throughput capacity of hybrid wireless 
networks where ad hoc nodes are randomly distributed and base stations are arbitrarily placed. They show that the pernode throughput capacity depends on the number of base stations, but the network still cannot scale.

Assuming $n$ nodes are randomly distributed and $m$ base stations are regularly placed, Liu et al. [16] study the throughput capacity of hybrid wireless networks. They consider two different routing strategies. Under $k$-nearest-cell routing strategy, if $m$ grows asymptotically slower than $\sqrt{n}$, the maximum per-node throughput capacity is $\Theta\left(\sqrt{\frac{1}{n \log n / m^{2}}} W\right)$, and the benefit of adding base stations is insignificant. However, if $m$ grows asymptotically faster than $\sqrt{n}$, the maximum per-node throughput capacity is $\Theta\left(\frac{m}{n} W\right)$, which increases linearly with the number of base stations. Under probabilistic routing strategy, similar results are obtained. The threshold of the number of base stations above which the per-node throughput capacity increases linearly with $m$ is $\sqrt{\frac{n}{\log n}}$. Thus, the network can scale if $m=\Omega(n)$.

Actually, the authors in [16] assume $k=0$, i.e., a node transmits to its destination in the ad hoc mode only if it is in the same cell. However, the 0-nearest-cell routing strategy cannot efficiently make use of the wireless channel. For example, if a source node and its destination are within onehop distance of each other, but they are not in the same cell, then they cannot directly communicate in the ad hoc mode according to the routing strategy. Instead, the transmissions between these two nodes can only be carried out through base stations. Besides, the case $k=0$ is too specific. To provide a solution to a more general case, and to better utilize the bandwidth resource, Pei et al. [23] propose to use the $L$-maximum-hop routing strategy. Unfortunately, the capacity bounds derived in [23] are not tight.

In this paper, we revisit the throughput capacity problem in hybrid wireless networks by using the $L$-maximum-hop resource allocation strategy in $[23]^{2}$. Specifically, a source node transmits to its destination in the ad hoc mode if the destination can be reached from the source within $L(L \geq 1)$ hops. Otherwise, the transmission will be carried out in the infrastructure mode. Assuming a total bandwidth of $W$ bits/sec is split into three parts, i.e., $W_{1}$ for ad hoc mode, $W_{2}$ for uplink in the infrastructure mode, and $W_{3}$ for downlink in the infrastructure mode, we show that:

1) when $L=\Omega\left(\frac{n^{\frac{1}{3}}}{\log ^{\frac{2}{3}} n}\right)$, the throughput capacity of the network is $\Theta\left(\frac{n W_{1}}{L \log n}\right)+\Theta\left(m W_{2}\right)$. If $m=\Omega\left(\frac{n}{L \log n}\right)$, we can have higher throughput when all the traffics are carried in the infrastructure mode. The per-node throughput capacity increases linearly with the number of base stations $m$, and the network can scale only if $m=\Omega(n)$. If $m=O\left(\frac{n}{L \log n}\right)$, we can achieve higher throughput when all the traffics are carried in the ad hoc mode, and the network cannot scale.

2) when $L=o\left(\frac{n^{\frac{1}{3}}}{\log \frac{2}{3} n}\right)$, the throughput capacity of the network is $\Theta\left(L^{2} \log n W_{1}\right)+\Theta\left(m W_{2}\right)$. If $m=$ $\Omega\left(L^{2} \log n\right)$, we can have higher throughput when all

\footnotetext{
${ }^{2}$ We call it a resource allocation strategy instead of a routing strategy because we only decide which kind of resource a transmission uses, and do not specify how to choose a route from a source to a destination.
}

the traffics are carried in the infrastructure mode. The per-node throughput capacity increases linearly with the number of base stations $m$, and the network can scale only if $m=\Omega(n)$. If $m=O\left(L^{2} \log n\right)$, we can achieve higher throughput when all the traffics are carried in the ad hoc mode, and the network cannot scale.

It can be easily shown that the results obtained in [16] under the 0-nearest-cell routing strategy is just a special case in 2) presented above. Furthermore, we also compare the throughput capacity of our hybrid wireless networks with that of pure ad hoc networks. The capacity gain is clearly shown.

In addition to the throughput capacity, the packet delay is also an important issue in the network. [2], [8], [10], [15], [19], and [20] propose to utilize nodes' mobility to deliver packets. Each packet is only relayed for very few times before arriving at the destination. For example, in [10], each packet is at most relayed once, i.e., relayed by at most one relaying node. They find that there is a trade-off between the capacity and the delay. Specifically, in pure ad hoc networks, the capacity can only be increased at the cost of greatly increased delay. Moreover, Gamal et al. [9] show that using mobility to increase throughput, even slightly, would lead to an abrupt and large increase in delay. In this paper, we also study the delay in hybrid wireless networks. We find that by adding base stations in pure ad hoc networks, the capacity can be improved without increasing the delay. Particularly, in hybrid wireless networks, the average packet delay can be maintained as low as $\Theta(1)$ even when the per-node throughput capacity is $\Theta(W)$.

The rest of this paper is organized as follows. In Section II we introduce some definitions. Section III gives the hybrid wireless network model, including the network architecture, the interference model, and the resource allocation strategy. In Section IV and Section V, we derive the throughput capacity and delay of hybrid wireless networks, respectively. We finally conclude this paper in Section VI.

\section{DEFINITIONS}

Throughput: As defined in the usual way, the time average of the number of bits per second that can be transmitted by each node to its destination is called the per-node throughput. The sum of per-node throughput over all the nodes in a network is called the throughput of the network.

Feasible Throughput: We say that the throughput of a network, denoted by $\lambda(n)$, is feasible if there exists a spatial and temporal scheduling scheme that yields an aggregate network throughput of $\lambda(n)$ bits/sec.

Throughput Capacity of A Network: We say that the throughput capacity of a network ( [14]) is of order $O(f(n))$ bits per second if there is a deterministic constant $c_{1}<+\infty$ such that

$$
\liminf _{n \rightarrow+\infty} \operatorname{Prob}\left(\lambda(n)=c_{1} f(n) \text { is feasible }\right)<1,
$$

and is of order $\Theta(f(n))$ bits per second if there are deterministic constants $0<c_{2}<c_{3}<+\infty$ such that

$$
\begin{aligned}
& \liminf _{n \rightarrow+\infty} \operatorname{Prob}\left(\lambda(n)=c_{2} f(n) \text { is feasible }\right)=1, \\
& \liminf _{n \rightarrow+\infty} \operatorname{Prob}\left(\lambda(n)=c_{3} f(n) \text { is feasible }\right)<1 .
\end{aligned}
$$


Average Packet Delay of A Network: The delay of a packet in a network is the time it takes the packet to reach the destination after it leaves the source. As in [15], [20], we do not consider the queuing delay at the source node since we are more interested in the network delay. The average packet delay of a network is obtained by averaging over all transmitted packets in the network. Besides, we also assume the packet size scales as the per-node throughput ${ }^{3}$.

\section{Hybrid WIRELESS NeTwORK MODEL}

\section{A. Network Architecture}

We consider a two-tier hybrid wireless network on the surface of a torus of unit area. The low tier is composed of $n$ normal nodes, and the higher tier consists of $m$ base stations, respectively. The assumption of a torus enables us to avoid technicalities arising out of edge effects, but the results derived in the paper are applicable for nodes located on an unit square as well.

We assume $n$ nodes are uniformly and independently distributed. They have the same transmission power, and hence the same transmission range denoted by $r(n)$. We follow the process in [10] to choose random sender-receiver pairs so that each node is a source node for one flow and a destination node for at most $O(1)$ flows.

The $m$ base stations are regularly placed in the network, dividing the area into a hexagonal tessellation, which is exactly the classical 7-cell reuse model as described in [24]. Each hexagon is called a cell and there is one base station in the center of each cell. Base stations do not serve as data sources or data destinations. Instead, they only help relay the packets for the normal nodes. Furthermore, we also assume base stations are inter-connected by an optical network, in which the link bandwidth is large enough. Thus, the wired network has no bandwidth constraints.

\section{B. Interference Model}

We employ the Protocol Model in [12] as the interference model. Suppose node $X_{i}$ transmits to another node $X_{j} . X_{i}$ and $X_{j}$ also denote the positions of these two nodes. Then, the transmission is successful if the following two conditions are satisfied:

1) The distance between $X_{i}$ and $X_{j}$ is no more than $r(n)$, the transmission range of the nodes, i.e.,

$$
\left|X_{i}-X_{j}\right| \leq r(n) \text {. }
$$

2) The positions of other transmitters $X_{k}$ simultaneously transmitting over the same channel should satisfy:

$$
\left|X_{k}-X_{j}\right| \geq(1+\Delta) r(n) \text {. }
$$

The quantity $\Delta>0$ models situations where a guard zone is specified by the protocol to prevent a neighboring node from transmitting on the same channel at the same time. It also allows for imprecision in the achieved range of transmissions.

\footnotetext{
${ }^{3}$ As pointed out in [9], under this assumption, queuing delay at source node can actually be a constant, which gives us another reason to focus on network delay.
}

\section{Resource Allocation Strategy}

In hybrid wireless networks, packets can be transmitted in two modes: ad hoc mode and infrastructure mode. In the ad hoc mode, packets are forwarded from the source to the destination with only the help of normal nodes, i.e., without the help of base stations. While in the infrastructure mode, packets are first transmitted from the source to the wired network, and then to the destination.

In this paper, we consider a L-maximum-hop $(L \geq 1)$ resource allocation strategy. In particular, if a destination node can be reached within $L$ hops from a source node, then the packets between this source and destination pair are transmitted in the ad hoc mode. Otherwise, packets are transmitted in the infrastructure mode.

Moreover, we assume a total bandwidth of $W$ bits $/ \mathrm{sec}^{4}$, which is split into three frequency bands, i.e., $W_{1}$ for ad hoc mode, $W_{2}$ for uplink for infrastructure mode, and $W_{3}$ for downlink for infrastructure mode, respectively. Since the uplink has the same amount of traffic as the downlink, we have $W_{2}=W_{3}$. Thus, $W=W_{1}+2 W_{2}$.

\section{CAPacity of Hybrid Wireless Networks Under $L$-MAXIMUM-HOP Resource AlLOCATION STRATEGY}

In this section, we derive the capacity of hybrid wireless networks under $L$-maximum-hop resource allocation strategy. We assume all nodes are equipped with omnidirectional antennas.

Recall that the transmissions in the ad hoc mode, the uplink and downlink transmissions in the infrastructure mode use different frequency bands, i.e., $W_{1}, W_{2}$, and $W_{3}$, respectively. We assume there is no interference between these three types of traffics, and they can be carried out simultaneously. Thus, the throughput capacity of the network with $n$ nodes and $m$ base stations, denoted by $\lambda(n, m)$, can be represented as

$$
\lambda(n, m)=\lambda_{a}(n, m)+\lambda_{i}(n, m)
$$

where $\lambda_{a}(n, m)$ and $\lambda_{i}(n, m)$ denote the throughput capacity contributed by the ad hoc mode transmissions and the infrastructure mode transmissions, respectively. Notice that $\lambda(n, m)$, $\lambda_{a}(n, m)$, and $\lambda_{i}(n, m)$ all denote the aggregated throughput capacity. We use $\lambda^{0}(n, m), \lambda_{a}^{0}(n, m)$, and $\lambda_{i}^{0}(n, m)$ to denote the corresponding per-node throughput capacity, respectively.

\section{A. Ad Hoc Mode Throughput Capacity}

Consider the low tier network component, i.e., $n$ uniformly and independently distributed nodes on a planar torus. We first introduce some of the definitions and results in [12], listed as follows. $c_{i}$ 's are used to denote deterministic constants independent of $n$.

Voronoi Tessellation [21]: Given a set of $n$ points in a plane, Voronoi tessellation divides the domain into a set of polygonal regions, the boundaries of which are the perpendicular bisectors of the lines joining the points.

\footnotetext{
${ }^{4}$ Notice that bandwith has several meanings. In signal processing, it is a measure of the width of a range of frequencies, measured in hertz. Here, however, we refer it to be a rate of data transfer, measured in bits per second.
} 
Lemma 4.1 in [12]: For every $\varepsilon>0$, there is a Voronoi tessellation with the property that every Voronoi cell contains a disk of radius $\varepsilon$ and is contained in a disk of radius $2 \varepsilon$.

Then for the $n$ nodes, we can construct a Voronoi tessellation $V_{n}$ for which

- (V1) Every Voronoi cell contains a disk of area $100 \log n / n$.

- (V2) Every Voronoi cell is contained in a disk of radius $2 \rho(n)$, where $\rho(n):=$ the radius of a disk of area $\frac{100 \log n}{n}$.

Adjacent Voronoi Cells: We say two Voronoi cells are adjacent if they share a common point (every Voronoi cell is a closed set).

We choose the range $r(n)$ of each transmission so that

$$
r(n)=8 \rho(n),
$$

which allows direct communication within a Voronoi cell and between adjacent Voronoi cells.

Interfering Neighbors: we say two cells are interfering neighbors if there is a point in one cell which is within a distance $(2+\Delta) r(n)$ of some point in the other cell.

Lemma 4.3 in [12]: When all nodes in the network use omnidirectional antennas, every cell in $V_{n}$ has no more than $c_{1}$ interfering neighbors, where $c_{1}$ depends only on $\Delta$ and grows no faster than linearly in $(1+\Delta)^{2}$.

Proof: Let $V$ be a Voronoi cell. If $V^{\prime}$ is an interfering neighboring Voronoi cell, there must be two points, one in $V$ and the other in $V^{\prime}$, which are no more than $(2+\Delta) r(n)$ units apart. From (V2), the diameter of a cell is bounded by $4 \rho(n)$. Hence $V^{\prime}$, and similarly every other interfering neighbor in the Protocol Model, must be contained within a common large disk $D$ of radius $6 \rho(n)+(2+\Delta) r(n)$. Such a disk cannot contain more than $c_{2}=\frac{[6 \rho(n)+(2+\Delta) r(n)]^{2}}{\rho^{2}(n)}=(22+8 \Delta)^{2} \sim$ $O\left((1+\Delta)^{2}\right)$ disks of radius $\rho(n)$. By (V1), there can therefore be no more than this number of cells within $D$. Thus, $c_{1}=$ $c_{2}-1$ is then an upper bound on the number of interfering neighbors of the cell.

Lemma 4.4 in [12]: In the Protocol Model, there is a schedule for transmitting packets such that in every $\left(1+c_{1}\right)$ slots, each cell in the tessellation $V_{n}$ gets one slot for packet transmission, and all transmissions are successfully received within a distance $r(n)$ from their transmitters.

We first derive a lower bound on the per-node throughput capacity by choosing the routes of packets to approximate the straightline connecting the source and the destination. Denote the straight line connecting a source node $X_{i}$ and a destination node $Y_{i}$ as $L_{i}$. Under the $L$-maximum-hop resource allocation strategy, we now bound the probability that $L_{i}$ intersects a given Voronoi cell $V$.

Lemma 1: For segment $L_{i}$ and Voronoi cell $V$, under the $L$-maximum-hop routing strategy,

$\operatorname{Prob}\left(L_{i}\right.$ intersects $V$ and $L_{i}$ is using $\left.W_{1}\right) \leq c_{3} L^{3}\left(\frac{\log n}{n}\right)^{2}$.

Proof: As mentioned before, Voronoi cell $V$ is contained in a disk of radius $2 \rho(n)$, i.e., $\sqrt{\frac{400 \log n}{\pi n}}$. Suppose $X_{i}$ lies at a distance $x$ from the center of this disk as shown in Fig. 1, then the angel $\alpha$ subtended at $X_{i}$ by the disk is no

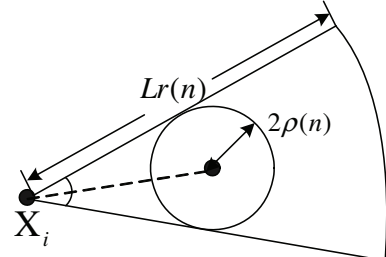

Fig. 1. Illustration for calculating the probability that $L_{i}$ intersects Voronoi cell $V$.

more than $\frac{c_{4}}{x} \sqrt{\frac{\log n}{n}}$. The area of the sector formed is no more than $\frac{c_{5} L^{2} r^{2}(n) \alpha}{2 \pi}$. If $Y_{i}$ does not lie in the sector, then the line $L_{i}$ cannot intersect the disk containing the cell $V$. Thus, the probability that $L_{i}$ intersects the disk is no more than $\frac{c_{6} L^{2}}{x} \cdot\left(\frac{\log n}{n}\right)^{\frac{3}{2}}$.

Since $X_{i}$ is uniformly distributed on the plane of unit disk, the probability density that it is at a distance $x$ from the center of the disk is bounded above by $2 c_{7} \pi x$. Besides, in order for $L_{i}$ to intersect $V$, we need $2 \rho(n) \leq x \leq \operatorname{Lr}(n)$. As a result, we can obtain

$$
\begin{aligned}
& \operatorname{Prob}\left(L_{i} \text { intersects } V \text { and } L_{i} \text { is using } W_{1}\right) \\
& \leq \int_{\sqrt{\frac{400 \log n}{\pi n}}}^{8 L \sqrt{\frac{100 \log n}{\pi n}}} \frac{c_{6} L^{2}}{x}\left(\frac{\log n}{n}\right)^{\frac{3}{2}} \cdot 2 c_{7} \pi x d x \\
& \leq c_{3} L^{3}\left(\frac{\log n}{n}\right)^{2} .
\end{aligned}
$$

Since there are $n$ lines $\left\{L_{i}\right\}_{i=1}^{n}$, connecting $X_{i}$ and $Y_{i}$, the mean number of lines passing through a Voronoi cell that use frequency band $W_{1}$ is bounded as follows:

$$
\begin{gathered}
E \text { (Number of lines in }\left\{L_{i}\right\}_{i=1}^{n} \text { intersects } V \\
\text { and } \left.L_{i} \text { is using } W_{1}\right) \leq c_{3} L^{3} \frac{\log ^{2} n}{n} .
\end{gathered}
$$

Notice that routes follow lines. By exploiting uniform convergence in the law of large numbers along the line in [12], we have the following two results.

Lemma 2: There is a $\delta^{\prime}(n) \rightarrow 0$ such that

$$
\begin{gathered}
\operatorname{Prob}\left(\operatorname { s u p } _ { V \in V _ { n } } \left(\text { Number of lines } L_{i} \text { intersecting } V \text { and } L_{i}\right.\right. \\
\left.\left.\quad \text { is using } W_{1}\right) \leq c_{3} L^{3} \frac{\log ^{2} n}{n}\right) \geq 1-\delta^{\prime}(n) .
\end{gathered}
$$

Note that the traffic handled by a cell is proportional to the number of lines passing through it. Since each line on frequency band $W_{1}$ carries traffic of rate $\lambda_{a}^{0}(n, m)$ bits per second, we have the following bound.

Lemma 3: There is a $\delta^{\prime}(n) \rightarrow 0$ such that

$$
\begin{aligned}
& \operatorname{Prob}\left(\sup _{V \in V_{n}}(\text { Traffic needing to be carried by cell } V \text { ) }\right. \\
& \left.\leq c_{3} \lambda_{a}^{0}(n, m) L^{3} \frac{\log ^{2} n}{n}\right) \geq 1-\delta^{\prime}(n) .
\end{aligned}
$$

This implies that the rate at which each cell needs to transmit is less than $c_{3} \lambda_{a}^{0}(n, m) L^{3} \frac{\log ^{2} n}{n}$ with high probability. This rate can be accommodated by all cells if it is less than the rate available, i.e., if

$$
c_{3} \lambda_{a}^{0}(n, m) L^{3} \frac{\log ^{2} n}{n} \leq \frac{W_{1}}{c_{2}} .
$$


Thus, we arrive at a lower bound on the per-node throughput capacity contributed by ad hoc mode transmissions, as shown in the following lemma.

Lemma 4: For ad hoc mode transmissions, under the $L$ maximum-hop resource allocation strategy,

1) when $L=\Omega\left(\frac{n^{\frac{1}{3}}}{\log \frac{2}{3} n}\right)$, there is a deterministic constant $c>0$ not depending on $n, \Delta$, or $W_{1}$, such that

$$
\lambda_{a}^{0}(n, m)=\frac{c n W_{1}}{(1+\Delta)^{2} L^{3} \log ^{2} n}
$$

bits per second is feasible with high probability, i.e.,

$$
\lambda_{a}^{0}(n, m)=\Omega\left(\frac{n W_{1}}{L^{3} \log ^{2} n}\right) .
$$

2) and when $L=o\left(\frac{n^{\frac{1}{3}}}{\log _{\frac{2}{3}} n}\right)$, there is a deterministic constant $c>0$ not depending on $n, \Delta$, or $W_{1}$, such that

$$
\lambda_{a}^{0}(n, m)=W_{1} .
$$

bits per second is feasible with high probability.

Next, we find an upper bound on the per-node throughput capacity.

Lemma 5.4 in [12]: The number of simultaneous transmissions on any particular channel is no more than

$$
N_{\max }=\frac{4}{c_{8} \pi \Delta^{2} r^{2}(n)}
$$

in the Protocol Model.

Under the $L$-maximum-hop resource allocation strategy, the mean number of hops taken by a packet transmitted in the ad hoc mode, denoted by $\bar{h}$, is calculated as follows:

$$
\begin{aligned}
\bar{h} \geq & 1 \cdot \frac{\pi r^{2}(n)}{\pi L^{2} r^{2}(n)}+2 \cdot \frac{3 \pi r^{2}(n)}{\pi L^{2} r^{2}(n)}+\ldots \\
& +L \frac{\left[L^{2}-(L-1)^{2}\right] \pi r^{2}(n)}{\pi L^{2} r^{2}(n)} \\
= & \frac{4 L^{3}+3 L^{2}-L}{6 L^{2}}
\end{aligned}
$$

Since each source generates $\lambda_{a}^{0}(n, m)$ bits per second, there are $n$ sources, each of which transmits to its destination in ad hoc mode with a probability of $\pi L^{2} r^{2}(n)$, then the total number of bits per second served by the entire network needs to be at least $n \pi L^{2} r^{2}(n) \bar{h} \lambda_{a}^{0}(n, m)$. To ensure that all the required traffic is carried, we therefore need

$$
n \pi L^{2} r^{2}(n) \bar{h} \lambda_{a}^{0}(n, m) \leq N_{\max } W_{1} .
$$

Thus,

$$
\lambda_{a}^{0}(n, m) \leq \frac{c_{9} W_{1}}{\Delta^{2} n L^{3} r^{4}(n)} .
$$

Since $r(n)>\sqrt{\frac{\log n}{\pi n}}$ is necessary to guarantee connectivity with high probability [11], then we obtain

$$
\lambda_{a}^{0}(n, m) \leq \frac{c^{\prime} n W_{1}}{\Delta^{2} L^{3} \log ^{2} n} .
$$

Besides, we also have $\lambda_{a}^{0}(n, m) \leq W_{1}$. Thus, we arrive at the following lemma.
Lemma 5: For ad hoc mode transmissions, under the $L$ maximum-hop resource allocation strategy,

1) when $L=\Omega\left(\frac{n^{\frac{1}{3}}}{\log ^{\frac{2}{3}} n}\right)$, an upper bound on per-node throughput capacity is

$$
\lambda_{a}^{0}(n, m)=\frac{c^{\prime} n W_{1}}{\Delta^{2} L^{3} \log ^{2} n}
$$

bits per second, where $c^{\prime}<+\infty$, not depending on $n$, $\Delta$, or $W_{1}$,

2) and when $L=o\left(\frac{n^{\frac{1}{3}}}{\log ^{\frac{2}{3}} n}\right)$, an upper bound on per-node throughput capacity is

$$
\lambda_{a}^{0}(n, m)=W_{1} .
$$

Notice that the probability that one node will transmit to its destination node in ad hoc mode is $\pi L^{2} r^{2}(n)$. Let $N_{i}(1 \leq$ $j \leq n$ ) be a random variable defined as follows:

$N_{i}= \begin{cases}1, & \text { source node } i \text { transmits to its destination node } \\ & \text { in ad hoc mode } \\ 0, & \text { otherwise }\end{cases}$

Let $N_{T}$ be a random variable defined as the total number of source nodes transmitting in ad hoc mode, i.e., $N_{T}=$ $\sum_{i=1}^{n} N_{i}$. Thus, the expected number source nodes in ad hoc mode is:

$$
E\left(N_{T}\right)=E\left(\sum_{i=1}^{n} N_{i}\right)=\sum_{i=1}^{n} E\left(N_{i}\right)
$$

Since $f\left(N_{i}=1\right)=\pi L^{2} r^{2}(n)$, and $r(n)$ needs to be $\Theta\left(\sqrt{\frac{\log n}{n}}\right)$ to make the network connected [11], we have ${ }^{5}$

$E\left(N_{i}\right)=1 \cdot \pi L^{2} r^{2}(n)+0 \cdot\left(1-\pi L^{2} r^{2}(n)\right)=\pi L^{2} \frac{\log n}{n}$. Thus,

$$
E\left(N_{T}\right)=n \cdot \pi L^{2} \frac{\log n}{n}=\pi L^{2} \log n .
$$

Recall the Chernoff bounds [5]:

- For any $\delta>0$,

$$
P\left[N_{T}>(1+\delta) \pi L^{2} \log n\right]<\left(\frac{e^{\delta}}{(1+\delta)^{1+\delta}}\right)^{\pi L^{2} \log n} .
$$

- For any $0<\delta<1$,

$$
P\left[N_{T}<(1-\delta) \pi L^{2} \log n\right]<e^{-\frac{1}{2} \delta^{2} \pi L^{2} \log n} .
$$

From the above, we can obtain for any $0<\delta<1$,

$$
P\left[\left|N_{T}-\pi L^{2} \log n\right|>\delta \pi L^{2} \log n\right]<e^{-\theta \pi L^{2} \log n} .
$$

where $\theta>0$. So, as $n \rightarrow \infty$, the total number of source nodes transmitting in ad hoc mode is equal to $\pi L^{2} \log n$ with probability 1 .

Thus, the total ad hoc mode traffic is $n \pi L^{2} r^{2}(n) \lambda_{a}^{0}(n, m)$, i.e., $c^{\prime \prime} L^{2} \log n W_{1}$. Combining Lemma 4 and Lemma 5 leads to the following theorem.

${ }^{5}$ Note $r(n)=\Theta\left(\sqrt{\frac{\log n}{n}}\right)$ means $r(n)=c_{r} \sqrt{\frac{\log n}{n}}$ where $0<c_{r}<$ $+\infty$. We ignore $c_{r}$ in the following derivations for simplicity, which will not change our final results. 
Theorem 1: Under the $L$-maximum-hop routing strategy, the throughput capacity of the network contributed by ad hoc mode transmissions is

$$
\lambda_{a}(n, m)= \begin{cases}\Theta\left(\frac{n W_{1}}{L \log n}\right), & L=\Omega\left(\frac{n^{\frac{1}{3}}}{\log ^{\frac{2}{3}} n}\right) ; \\ \Theta\left(\left(L^{2} \log n\right) W_{1}\right), & L=o\left(\frac{n^{\frac{1}{3}}}{\log ^{\frac{2}{3}} n}\right) .\end{cases}
$$

\section{B. Infrastructure Mode Throughput Capacity}

We then derive the throughput capacity contributed by transmissions in the infrastructure mode. Notice that each packet transmitted from a source to its destination in infrastructure mode will use one uplink and one downlink, and hence it should be counted only once for the throughput capacity.

Since the bandwidth for uplink is $W_{2}$ bits/sec, the throughput capacity per cell, denoted by $\lambda_{i}^{c}(n, m)$, is upper bounded by $W_{2}$. As we mentioned before, the base stations divide the area into a hexagon tessellation, i.e., a 7-cell frequency reuse pattern. Thus, the throughput capacity per cell is lower bounded by $\frac{1}{7} W_{2}$. Finally, we obtain the following theorem.

Theorem 2: Under the $L$-maximum-hop routing strategy, the throughput capacity of the network contributed by infrastructure mode transmissions is

$$
\lambda_{i}(n, m)=\Theta\left(m W_{2}\right) .
$$

Proof: We have shown that $\lambda_{i}^{c}(n, m)=\Theta\left(W_{2}\right)$. There are $m$ cells, which leads to $\lambda_{i}(n, m)=\Theta\left(m W_{2}\right)$.

\section{Throughput Capacity of the Network}

From Theorem 1 and Theorem 2, we can obtain the following theorem.

Theorem 3: Under the $L$-maximum-hop resource allocation strategy, the throughput capacity of the network is

$$
\lambda_{a}(n, m)= \begin{cases}\Theta\left(\frac{n W_{1}}{L \log n}\right)+\Theta\left(m W_{2}\right), & L=\Omega\left(\frac{n^{\frac{1}{3}}}{\log _{\frac{1}{3}}^{2} n}\right) ; \\ \Theta\left(L^{2} \log n W_{1}\right)+\Theta\left(m W_{2}\right), & L=o\left(\frac{n^{\frac{1}{3}}}{\log ^{\frac{2}{3}} n}\right) .\end{cases}
$$

Case 1: $L=\Omega\left(\frac{n^{\frac{1}{3}}}{\log ^{\frac{2}{3}} n}\right)$.

According to Theorem 3, we have

$$
\lambda(n, m)=\Theta\left(\frac{n W_{1}}{L \log n}\right)+\Theta\left(m W_{2}\right) .
$$

- If $m=\Omega\left(\frac{n}{L \log n}\right)$, then we can have higher throughput when $W_{1}=0$, i.e., $W_{2}=W / 2$, and

$$
\lambda_{\max }(n, m)=\Theta(m W),
$$

and hence,

$$
\lambda_{\text {max }}^{0}(n, m)= \begin{cases}\Theta(W), & \text { if } m=\Omega(n) ; \\ \Theta\left(\frac{m W}{n}\right), & \text { if } m=o(n) .\end{cases}
$$

- If $m=o\left(\frac{n}{L \log n}\right)$, then we can have higher throughput when $W_{2}=0$, i.e., $W_{1}=W$, and

$$
\lambda_{\max }(n, m)=\Theta\left(\frac{n W}{L \log n}\right),
$$

and hence,

$$
\lambda_{\max }^{0}(n, m)=\Theta\left(\frac{W}{L \log n}\right) .
$$

Since $L=\Omega\left(\frac{n^{\frac{1}{3}}}{\log ^{\frac{2}{3}} n}\right)$, then $L \log n \rightarrow \infty$, and hence $\lambda_{\text {max }}^{0}(n, m) \rightarrow 0$ as $n \rightarrow \infty$, which means the per-node throughput capacity diminishes as $n$ goes large and the network cannot scale.

Case 2: $L=o\left(\frac{n^{\frac{1}{3}}}{\log ^{\frac{2}{3}} n}\right)$.

According to Theorem 3, we have

$$
\lambda(n, m)=\Theta\left(L^{2} \log n W_{1}\right)+\Theta\left(m W_{2}\right) .
$$

- If $m=\Omega\left(L^{2} \log n\right)$, then we can have higher throughput when $W_{1}=0$, i.e., $W_{2}=W / 2$, and

$$
\lambda_{\max }(n, m)=\Theta(m W),
$$

and hence,

$$
\lambda_{\text {max }}^{0}(n, m)= \begin{cases}\Theta(W), & \text { if } m=\Omega(n) ; \\ \Theta\left(\frac{m W}{n}\right), & \text { if } m=o(n) .\end{cases}
$$

- If $m=o\left(L^{2} \log n\right)$, then we can have higher throughput when $W_{2}=0$, i.e., $W_{1}=W$, and

$$
\lambda_{\max }(n, m)=\Theta\left(L^{2} \log n W\right),
$$

and hence,

$$
\lambda_{\max }^{0}(n, m)=\Theta\left(\frac{L^{2} \log n}{n} W\right) .
$$

Since $L=o\left(\frac{n^{\frac{1}{3}}}{\log ^{\frac{2}{3}} n}\right)$, then $\frac{L^{2} \log n}{n} \rightarrow 0$ as $n \rightarrow \infty$, which means the per-node throughput capacity diminishes as $n$ goes large and the network cannot scale.

From the above, we arrive at the following results.

Corollary 1: Under the $L$-maximum-hop resource allocation strategy,

1) when $L=\Omega\left(\frac{n^{\frac{1}{3}}}{\log ^{\frac{2}{3}} n}\right)$, (i) if $m=\Omega\left(\frac{n}{L \log n}\right)$, we can have higher throughput when $W_{1}=0$, and the network can scale only if $m=\Omega(n)$; (ii) if $m=o\left(\frac{n}{L \log n}\right)$, we can have higher throughput when $W_{2}=0$, and the network cannot scale.

2) when $L=o\left(\frac{n^{\frac{1}{3}}}{\log ^{\frac{2}{3}} n}\right)$, (i) if $m=\Omega\left(L^{2} \log n\right)$, we can have higher throughput when $W_{1}=0$, and the network can scale only if $m=\Omega(n)$; (ii) if $m=o\left(L^{2} \log n\right)$, we can have higher throughput when $W_{2}=0$, and the network cannot scale.

\section{Comparisons with Pure Ad Hoc Networks}

Gupta and Kumar have shown in [12] that for pure ad hoc networks, when each node randomly chooses another node as its destination with no limit to the maximum number of hops, the per-node throughput capacity is $\Theta\left(\frac{W}{\sqrt{n \log n}}\right)$. We discuss in the following whether the per-node throughput capacity can be enhanced by placing some base stations in the network and the impacts of $L$ and $m$ on the throughput capacity of hybrid wireless networks.

Case 1: $L=\Omega\left(\frac{n^{\frac{1}{3}}}{\log ^{\frac{2}{3}} n}\right)$.

1) If $m=\Omega\left(\frac{n}{L \log n}\right)$,

- If $m=\Omega(n)$, then $\lambda_{\text {max }}^{0}(n, m)=\Theta(W)$.

- If $m=o(n)$, then $\lambda_{\max }^{0}(n, m)=\Theta\left(\frac{m W}{n}\right)=$ $\Omega\left(\frac{W}{L \log n}\right)$. Since the transmission range of the 
nodes satisfies $r(n)>\sqrt{\frac{\log n}{\pi n}}$ as mentioned before, then $L=O\left(\sqrt{\frac{n}{\log n}}\right)$. Thus, we obtain $\lambda_{\max }^{0}(n, m)=\Omega\left(\frac{W}{\sqrt{n \log n}}\right)$. Moreover, when $L=\Theta\left(\frac{n^{\frac{1}{3}}}{\log ^{\frac{2}{3}} n}\right)$, we can obtain $\lambda_{\max }^{0}(n, m)=$ $\Omega\left(\frac{W}{n^{\frac{1}{3}} \log \frac{1}{3} n}\right)$, and when $L=\omega\left(\frac{n^{\frac{1}{3}}}{\log ^{\frac{2}{3}} n}\right)$, we can obtain $\lambda_{\max }^{0}(n, m)=\Omega\left(o\left(\frac{W}{n^{\frac{1}{3}} \log ^{\frac{1}{3}} n}\right)\right)$.

2) If $m=o\left(\frac{n}{L \log n}\right)$, we have shown that $\lambda_{\max }^{0}(n, m)=$ $\Theta\left(\frac{W}{L \log n}\right)$. Since $L=O\left(\sqrt{\frac{n}{\log n}}\right)$, we have $\lambda_{\max }^{0}(n, m)=\Omega\left(\frac{W}{\sqrt{n \log n}}\right)$. Furthermore, when $L=$ $\Theta\left(\frac{n^{\frac{1}{3}}}{\log \frac{2}{3} n}\right)$, we have $\lambda_{\max }^{0}(n, m)=\Theta\left(\frac{W}{n^{\frac{1}{3}} \log ^{\frac{1}{3}} n}\right)$, and when $L=\omega\left(\frac{n^{\frac{1}{3}}}{\log ^{\frac{2}{3}} n}\right)$, we have $\lambda_{\max }^{0}(n, m)=$ $o\left(\frac{W}{n^{\frac{1}{3}} \log \frac{1}{3} n}\right)$.

In this case, we limit $L$ to $\Omega\left(\frac{n^{\frac{1}{3}}}{\log \frac{2}{3} n}\right)$. If $m=o\left(\frac{n}{L \log n}\right)$, the increase of base stations does not increase the per-node throughput capacity. But, the throughput capacity of hybrid wireless networks is greater than that of pure ad hoc networks. If we add some more base stations in the network so that $m$ is lower bounded by $\frac{n}{L \log n}$ but upper bounded by $n$, the per-node throughput capacity increases linearly with the number of base stations, which is $\Theta\left(\frac{m W}{n}\right)$, and also greater than that of pure ad hoc networks. Moreover, if we keep adding base stations in the network such that $m=\Omega(n)$, the per-node throughput capacity will reach its maximum, i.e., $\Theta(W)$. Besides, we also observe that except for the case that $m=\Omega(n)$, the throughput capacity of hybrid wireless networks always gets smaller as the maximum number of hops $L$ increases.

Case 2: $L=o\left(\frac{n^{\frac{1}{3}}}{\log ^{\frac{2}{3}} n}\right)$.

We notice that in this case, $L^{2} \log n=o\left(\frac{n^{\frac{2}{3}}}{\log ^{\frac{1}{3}} n}\right)$.

1) If $m=\Omega\left(L^{2} \log n\right)$,

- If $m=\Omega(n)$, we have shown that $\lambda_{\max }^{0}(n, m)=$ $\Theta(W)$.

- If $m=o(n), \lambda_{\max }^{0}(n, m)=\Theta\left(\frac{m W}{n}\right)$. Thus, if $m=\Omega\left(\frac{n^{\frac{2}{3}}}{\log ^{\frac{1}{3}} n}\right)$, then $\lambda_{\max }^{0}(n, m)=\Omega\left(\frac{W}{n^{\frac{1}{3}} \log ^{\frac{1}{3}} n}\right)$; and if $m=o\left(\frac{n^{\frac{2}{3}}}{\log ^{\frac{1}{3}} n}\right)$, then $\lambda_{\max }^{0}(n, m)=$ $o\left(\frac{W}{n^{\frac{1}{3}} \log ^{\frac{1}{3}} n}\right)$. Furthermore, when $L=\Omega\left(\frac{n^{\frac{1}{4}}}{\log ^{\frac{3}{4}} n}\right)$, we obtain that $\lambda_{\max }^{0}(n, m)=\Omega\left(\frac{W}{\sqrt{n \log n}}\right)$, and when $L=o\left(\frac{n^{\frac{1}{4}}}{\log ^{\frac{3}{4}} n}\right)$, we obtain that $\lambda_{\max }^{0}(n, m)=$ $\Omega\left(o\left(\frac{W}{\sqrt{n \log n}}\right)\right)$.

2) If $m=o\left(L^{2} \log n\right)=o\left(\frac{n^{\frac{2}{3}}}{\log ^{\frac{1}{3}} n}\right)$, then $\lambda_{\max }^{0}(n, m)=$ $\Theta\left(\frac{L^{2} \log n}{n} W\right)=o\left(\frac{W}{n^{\frac{1}{3}} \log ^{\frac{1}{3}} n}\right)$. Moreover, when $L=$ $\Omega\left(\frac{n^{\frac{1}{4}}}{\log \frac{3}{4} n}\right)$, we obtain that $\lambda_{\max }^{0}(n, m)=\Omega\left(\frac{W}{\sqrt{n \log n}}\right)$, and when $L=o\left(\frac{n^{\frac{1}{4}}}{\log ^{\frac{3}{4}} n}\right)$, we obtain that $\lambda_{\max }^{0}(n, m)=$ $o\left(\frac{W}{\sqrt{n \log n}}\right)$.
In this case, we further limit $L$ to $o\left(\frac{n^{\frac{1}{3}}}{\log \frac{2}{3} n}\right)$. If $m=$ $o\left(L^{2} \log n\right)$, the increase of base stations does not increase the per-node throughput capacity, which is $o\left(\frac{W}{n^{\frac{1}{3}} \log ^{\frac{1}{3}} n}\right)$. If we add some more base stations in the network so that $m=\Omega\left(L^{2} \log n\right)$, the per-node throughput capacity increases linearly with the number of base stations. If $m=\Omega\left(\frac{n^{\frac{2}{3}}}{\log ^{\frac{1}{3}} n}\right)$, then the per-node throughput capacity will be lower bounded by $\frac{W}{n^{\frac{1}{3}} \log ^{\frac{1}{3}} n}$. If $m=\Omega(n)$, the per-node throughput capacity will reach its maximum, i.e., $\Theta(W)$. Besides, we observe that except for the case that $m=\Omega(n)$, the throughput capacity of hybrid wireless networks gets larger as the maximum number of hops $L$ increases, which is quite different from that in Case 1. Notice that when $L$ is as small as $o\left(\frac{n^{\frac{1}{4}}}{\log ^{\frac{3}{4}} n}\right)$, the capacity of hybrid wireless networks can be even smaller than that of pure ad hoc networks. This is because in this case too many nodes share the resource in the infrastructure mode, which significantly limits the network capacity.

From the above, we also find that adding base stations into the pure ad hoc networks can have significant impacts on the network capacity only if $m=\Omega\left(\frac{n}{L \log n}\right)$ when $L=\Omega\left(\frac{n^{\frac{1}{3}}}{\log ^{\frac{2}{3}} n}\right)$, or $m=\Omega\left(L^{2} \log n\right)$ when $L=o\left(\frac{n^{\frac{1}{3}}}{\log ^{\frac{2}{3}} n}\right)$. In other words, as the maximum hop count $L$ increases, the threshold of $m$, above which the capacity of hybrid wireless networks increases linearly with $m$, first increases when $L$ is small, and then decreases when $L$ is large.

\section{E. More Discussions}

In [16], the authors use a routing strategy such that a source node transmits to its destination node in the ad hoc mode only if the destination is in the same cell as the source. They show that under this routing strategy, the maximum throughput capacity increases linearly with the number of base stations $m$ if $m=\Omega(\sqrt{n})$. We show in the following that this can be considered as a special case in our analysis.

When $m=\Omega(\sqrt{n})$, we can obtain that $L=$ $O\left(\frac{1}{\sqrt{m}} / r(n)\right)=O\left(\frac{n^{\frac{1}{4}}}{\sqrt{\log n}}\right)$, and hence $m=\Omega\left(L^{2} \log n\right)$. Actually, this is included in the case that $L=o\left(\frac{n^{\frac{1}{3}}}{\log ^{\frac{2}{3}} n}\right)$ as we have shown before. According to our results, when $m=\Omega(\sqrt{n})=\Omega\left(L^{2} \log n\right)$, the maximum throughput capacity increases linearly with $m$, which is the same as that in [16].

\section{DELAY IN HYBRID WIRELESS NETWORKS UNDER $L$-MAXIMUM-HOP RESOURCE Allocation Strategy}

In the literature, there are some works ( [2], [8], [9], [15], [19], [20]) about the trade-off between capacity and delay in mobile ad hoc networks. They show that by using mobility to increase the capacity of the network, the delay will also be increased. Recall that in static random ad hoc networks, the per-node throughput capacity is $\Theta\left(\frac{1}{\sqrt{n \log n}}\right)$, and the average packet delay is $\Theta\left(\sqrt{\frac{n}{\log n}}\right)$. Gammal et al. [9] show that when the capacity of mobile random ad hoc networks increases to $\Theta(1)$, the average packet delay increases to $\Theta(n \log n)$. In this 
TABLE I

Throughput CAPacity and Packet Delay in Hybrid Wireless Networks.

\begin{tabular}{|c|c|c|c|}
\hline$L$ & $m$ & Per-node Throughput Capacity & Average Packet Delay \\
\hline \multirow{3}{*}{$\Omega\left(\frac{n^{\frac{1}{3}}}{\log \frac{2}{3} n}\right)$} & $\Omega(n)$ & $\Theta(W)$ & \multirow{2}{*}{$\Theta(1)$} \\
\cline { 2 - 3 } & $\left(\Omega\left(\frac{n}{L \log n}\right), o(n)\right)$ & $\Theta\left(\frac{m W}{n}\right)$ & $\Theta(L)$ \\
\cline { 2 - 3 } & $o\left(\frac{n}{L \log n}\right)$ & $\Theta\left(\frac{W}{L \log n}\right)$ & \multirow{2}{*}{$\Theta(1)$} \\
\hline \multirow{3}{*}{$o\left(\frac{n^{\frac{1}{3}}}{\log \frac{2}{3} n}\right)$} & $\Omega(n)$ & $\Theta(W)$ & $\Theta(L)$ \\
\cline { 2 - 3 } & $\left(\Omega\left(L^{2} \log n\right), o(n)\right)$ & $\Theta\left(\frac{m W}{n}\right)$ & $\Theta\left(\frac{L^{2} \log n}{n} W\right)$ \\
\cline { 2 - 3 } & $o\left(L^{2} \log n\right)$ & & \\
\hline
\end{tabular}

section, we study the delay in hybrid wireless networks to see whether there also exists such a trade-off.

We first present a fundamental result, which will be used frequently, as follows.

Lemma 6: Under $L$-maximum-hop resource allocation strategy, if we choose the route of packets to approximate the straightline connecting the source and the destination, for packets transmitted in the ad hoc mode, the average delay is $\Theta(L)$, and for packets transmitted in the infrastructure mode, the average delay is $\Theta(1)$.

Recall that the number of transmitters in the ad hoc mode is $\pi L^{2} \log n$ with probability 1 . Then, the number of transmitters in the infrastructure mode is $n-\pi L^{2} \log n$ with probability 1. Thus, the average delay of all the packets in the hybrid network, denoted by $D^{0}(n)$, is

$$
\begin{aligned}
D^{0}(n) & =\Theta\left(\frac{\pi L^{2} \log n \cdot L+\left(n-\pi L^{2} \log n\right) \cdot 1}{n}\right) \\
& =\Theta\left(\frac{L^{3} \log n}{n}+1\right) .
\end{aligned}
$$

Case 1: $L=\Omega\left(\frac{n^{\frac{1}{3}}}{\log ^{\frac{2}{3}} n}\right)$.

As we mentioned before, the average delay of all the packets in the hybrid network is

$$
D^{0}(n)=\Theta\left(\frac{L^{3} \log n}{n}+1\right)=\Omega(1) .
$$

1) If $m=\Omega\left(\frac{n}{L \log n}\right)$, we can have higher throughput when all the bandwidth is assigned to the traffics in the infrastructure mode, i.e., there are no traffics in the ad hoc mode. Thus, the average delay of all the packets is

$$
D_{\max }^{0}(n)=\Theta(1)
$$

2) $m=o\left(\frac{n}{L \log n}\right)$, we can have higher throughput when all the bandwidth is assigned to the ad mode traffics. In this case, the average delay of all the packets is

$$
D_{\max }^{0}(n)=\Theta(L)=\Omega\left(\frac{n^{\frac{1}{3}}}{\log ^{\frac{2}{3}} n}\right) .
$$

Besides, since $L=O\left(\sqrt{\frac{n}{\log n}}\right)$, we also have $D_{\text {max }}^{0}(n)=O\left(\sqrt{\frac{n}{\log n}}\right)$.

Thus, we observe that in this case, as we put more and more base stations in the network so that $m$ increases from $o\left(\frac{n}{L \log n}\right)$ to $\Omega\left(\frac{n}{L \log n}\right)$, the delay decreases while the capacity increases as we show in Section IV-D.

Case 2: $L=o\left(\frac{n^{\frac{1}{3}}}{\log ^{\frac{2}{3}} n}\right)$.
The same as before, the average delay of all the packets in the hybrid network is

$$
D^{0}(n)=\Theta\left(\frac{L^{3} \log n}{n}+1\right)=\Theta(1) .
$$

1) If $m=\Omega\left(L^{2} \log n\right)$, we can have higher throughput when all the bandwidth is assigned to the traffics in the infrastructure mode, and the average delay of all the packets is

$$
D_{\max }^{0}(n)=\Theta(1) .
$$

2) $m=o\left(L^{2} \log n\right)$, we can have higher throughput when all the bandwidth is assigned to the ad mode traffics, and the average delay of all the packets is

$$
D_{\text {max }}^{0}(n)=\Theta(L)=o\left(\frac{n^{\frac{1}{3}}}{\log ^{\frac{2}{3}} n}\right) .
$$

Besides, since $L=\Omega(1)$, we have

$$
D_{\text {max }}^{0}(n)=\Theta(L)=\Omega(1) \text {. }
$$

Similar to that when $L=\Theta\left(\frac{n^{\frac{1}{3}}}{\log \frac{2}{3} n}\right)$, as $m$ increases from $o\left(L^{2} \log n\right)$ to $\Omega\left(L^{2} \log n\right)$, the delay decreases while the capacity increases.

In conclusion, we find that the smaller the maximum hop number $L$ is, the smaller the average packet delay is. Specifically, when $L=\Omega\left(\frac{n^{\frac{1}{3}}}{\log ^{\frac{2}{3}} n}\right)$, the average delay is lower bounded by $\frac{n^{\frac{1}{3}}}{\log ^{\frac{2}{3}} n}$ if $m=o\left(\frac{n}{L \log n}\right)$, and is $\Theta(1)$ if $m=\Omega\left(\frac{n}{L \log n}\right)$. Besides, when $L=o\left(\frac{n^{\frac{1}{3}}}{\log ^{\frac{2}{3}} n}\right)$, the delay is $o\left(\frac{n^{\frac{1}{3}}}{\log \frac{2}{3} n}\right)$ if $m=o\left(L^{2} \log n\right)$, and is $\Theta(1)$ if $m=\Omega\left(L^{2} \log n\right)$.

Combining the results of the throughput capacity and the corresponding delay, we arrive at Table I, from which we observe that in hybrid wireless networks, by adding base stations to help carry out transmissions, the per-node throughput capacity can achieve $\Theta(W)$ while the average packet delay is kept as low as $\Theta(1)$.

\section{CONCLUSiON}

In this paper, we study the throughput capacity and the average packet delay in hybrid wireless networks. We find that for most of the cases, hybrid wireless networks have greater throughput capacity and smaller average packet delay than pure ad hoc networks. Moreover, we observe that when $m=$ $\Omega(n)$, the per-node throughput capacity can be $\Theta(W)$ while the average packet delay is maintained as low as $\Theta(1)$. Only 
when $L=o\left(\frac{n^{\frac{1}{4}}}{\log _{\frac{3}{4}}^{\frac{3}{4}} n}\right)$ and $m=o\left(L^{2} \log n\right)$, hybrid wireless networks have smaller throughput capacity than pure ad hoc networks. This is because in this case, there are a small number of base stations while too many nodes share the bandwidth in the infrastructure mode.

We also notice that we need to assign all the bandwidth to either ad hoc mode transmissions or infrastructure mode transmissions in order to have higher throughput. In either case, one of the two mode transmissions will get no bandwidth at all. In order to avoid this situation, we can assign some minimum amount of bandwidth to each mode, as suggested in [16].

Since hybrid wireless networks can provide high throughput capacity and low packet delay, we can finally conclude that wireless hybrid networks is a good solution to broadband access networks.

\section{REFERENCES}

[1] A. Agarwal and P. Kumar. Capacity bounds for ad hoc and hybrid wireless networks. ACM SIGCOMM Computer Communication Review, 34(3):71-81, July 2004.

[2] N. Bansal and Z. Liu. Capacity, delay, and mobility in wireless adhoc networks. In Proceeding of the IEEE International Conference on Computer Communications (INFOCOM'03), San Francisco, California, USA, March 2003.

[3] L. Braten, I. Tardy, A. Nordbotten, Z. Elek, and A. Morozova. Future broadband access networks. http://www.wibrace.org/ broadbandtutorials.php.

[4] C. Buraagohain, S. Suri, C. Toth, and Y. Zhou. Improved throughput bounds for interference-aware routing in wireless networks. In $\mathrm{CO}$ COON'07, Banff, Canada, July 2007.

[5] T. Cormen, C. Leiserson, R. Rivest, and C. Stein. Introduction to Algorithms (2nd ed.). MIT Press, 2001.

[6] O. Dousse, M. Franceschetti, and P. Thiran. On the throughput scaling of wireless relay networks. IEEE Trans. Inform. Theory (Joint special issue with IEEE/ACM Trans. Networking), 52(6):2756-2761, 2006.

[7] E. Duarte-Melo, A. Josan, M. Liu, D. Neuhoff, and S. Pradhan. The effect of node density and propagation model on throughput scaling of wireless networks. In Proc. IEEE International Symposium on Information Theory (ISIT), July 2006.

[8] A. Gamal, J. Mammen, B. Prabhakar, and D. Shah. Throughput-delay trade-off in wireless networks. In Proc. IEEE International Conference on Computer Communications (INFOCOM'04), Hong Kong, China, March 2004.

[9] A. Gamal, J. Mammen, B. Prabhakar, and D. Shah. Throughput-delay trade-off in wireless networks - part i: The fluid model. IEEE Trans. Inform. Theory, 52(6):2568-2592, 2006.

[10] M. Grossglauser and D. Tse. Mobility increases the capacity of ad hoc wireless networks. IEEE/ACM Trans. Networking, 10(4):477-486, August 2002.

[11] P. Gupta and P. Kumar. Critical power for asymptotic connectivity in wireless networks. Stochastic Analysis, Control, Optimization and Applications, A Volume in Honor of W.H. Fleming:547-566, 1998.

[12] P. Gupta and P. Kumar. The capacity of wireless networks. IEEE Trans. Inform. Theory, 46(2):388-404, March 2000.

[13] D. Knuth. The Art of Computer Programming. Addison-Wesley, 1998.

[14] U. Kozat and L. Tassiulas. Throughput capacity of random ad hoc networks with infrastructure support. In Proc. ACM MobiCom, Annapolis, MD, USA, June 2003.

[15] X. Lin, G. Sharma, R. Mazumdar, and N. Shroff. Degenerate delay-capacity tradeoffs in ad-hoc networks with brownian mobility. IEEE/ACM Trans. Networking, Special Issue on Networking and Information Theory, 14:2777-2784, 2006.

[16] B. Liu, Z. Liu, and D. Towsley. On the capacity of hybrid wireless networks. In Proc. IEEE International Conference on Computer Communications (INFOCOM'03), San Francisco, California, USA, March 2003

[17] B. Liu, P. Thiran, and D. Towsley. Capacity of a wireless ad hoc network with infrastructure. In Proc. ACM MobiHoc, Montreal, Quebec, Canada, September 2007.
[18] H. Luo, R. Ramjee, P. Sinha, L. Li, and S. Lu. Ucan: a unified cellular and ad-hoc network architecture. In Proc. ACM MobiCom, San Diego, California, USA, September 2003.

[19] J. Mammen and D. Shah. Throughput and delay in random wireless networks with restricted mobility. IEEE Trans. Information Theory, 53(3):1108-1116, 2007.

[20] R. Moraes, H. Sadjadpour, and J. Garcia-Luna-Aceves. On mobilitycapacity-delay trade-off in wireless ad hoc networks. In Proceeding of the IEEE/ACM International symposium on Modeling, Analysis, and Simulation of Computer and Telecommunication Systems (MASCOTS'04), Volendam, Netherlands, October 2004.

[21] A. Okabe, B. Boots, K. Sugihara, and S. Chiu. Spatial tessellations : concepts and applications of Voronoi diagrams. Wiley Series in Probability and Statistics, 2000.

[22] A. Ozgur, O. Leveque, and D. Tse. How does the information capacity of ad hoc networks scale? In Proc. Forty-fourth Annual Allerton Conference on Communication, Control and Computing, Monticello, IL, USA, September 2006.

[23] Y. Pei, J. Modestino, and X. Wang. On the throughput capacity of hybrid wireless networks using an l-maximum-hop routing strategy. In Proc. IEEE Vehicular Technology Conference, 2003.

[24] T. Rappaport. Wireless Communications: Principles and Practice (Second Edition). Prentice-Hall PTR, 2002.

[25] S. Sarkar, S. Dixit, and B. Mukherjee. An evolution scenario of a broadband access network using r-soa-based wdm-pon technologies. $J$. Lightwave Technol., 25(11):3479-3487, 2007.

[26] S. Sarkar, S. Dixit, and B. Mukherjee. Hybrid wireless-optical broadband-access network (woban): A review of relevant challenges. J. Lightwave Technol., 25(11):3329-3340, 2007.

[27] S. Toumpis. Capacity bounds for three classes of wireless networks. In Proc. ACM MobiHoc, Roppongi Hills, Tokyo, Japan, May 2004.

[28] A. Zemlianov and G. Veciana. Capacity of ad hoc wireless networks with infrastructure support. IEEE J. Sel. Areas Commun., 23(3), March 2005.

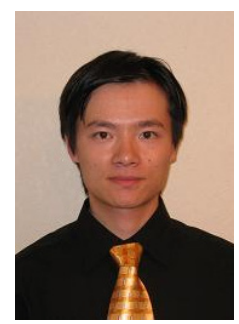

Pan Li (IEEE, S'06 / ACM'07) received his B.E. in Electrical Engineering from Huazhong University of Science and Technology, Wuhan, China, in 2005. $\mathrm{He}$ is working towards his Ph.D. degree in the Department of Electrical and Computer Engineering at University of Florida. His research interests include capacity and connectivity analysis, medium access control, routing algorithms, and cross-layer design in wireless networks. He is a student member of the IEEE and the ACM

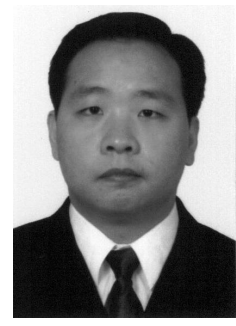

Chi Zhang (IEEE, S'06) received the B.E and M.E. degrees in Electrical Engineering from Huazhong University of Science and Technology, Wuhan, China, in July 1999 and January 2002, respectively. Since September 2004, he has been working towards the Ph.D. degree in the Department of Electrical and Computer Engineering at University of Florida, Gainesville, Florida, USA. His research interests are network and distributed system security, wireless networking, and mobile computing

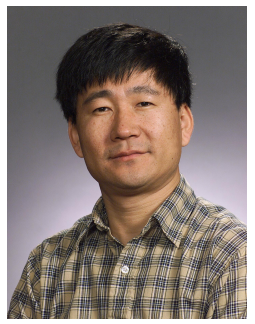

Yuguang "Michael" Fang (IEEE, S'92-M'94S'96-M'97-SM'99-F'08) received a Ph.D. degree in Systems Engineering from Case Western Reserve University in January 1994 and a Ph.D degree in Electrical Engineering from Boston University in May 1997. He was an assistant professor in the Department of Electrical and Computer Engineering at New Jersey Institute of Technology from July 1998 to May 2000. He then joined the Department of Electrical and Computer Engineering at University of Florida in May 2000 as an assistant professor, got an early promotion to an associate professor with tenure in August 2003 and to a full professor in August 2005. He holds a University of Florida Research Foundation (UFRF) Professorship from 2006 to 2009. 